\title{
Escala de Avaliação de Sintomas-40 (EAS-40): validade e precisão em amostra não-clínica
}

\author{
Validade e precisão da EAS-40 \\ Elisa Medici Pizão Yoshida \\ Fernanda Robert de Carvalho Santos Silva
}

\section{Resumo}

\begin{abstract}
A EAS-40, uma escala de 40 itens, de tipo auto-relato, visa avaliar sintomas psicopatológicos e foi adaptada do Symptom Checklist-90-R (SCL-90-R) para pacientes ambulatoriais brasileiros de hospitais gerais. $O$ objetivo da pesquisa foi estimar a validade de grupos comparados, validade concorrente e precisão de teste e reteste da EAS-40 para população não-clínica. A amostra de 113 universitários $(99 \mathrm{M}$ e $14 \mathrm{H})$ respondeu a EAS-40 e à versão brasileira da Toronto Alexithymia Scale (TAS) (critério externo). Os resultados sugerem que a EAS-40 pode ser útil para a avaliação de sintomas psicológicos de universitários com boa estabilidade temporal, permitindo discriminar populações com diferentes perfis. Quanto à validade concorrente, as dimensões da EAS-40 mostraram-se correlacionadas de formas diversas com os componentes da TAS. Novas pesquisas devem ser realizadas, envolvendo uma melhor distribuição das amostras quanto ao sexo.
\end{abstract}

Palavras-chave: Psicoticismo; ansiedade; somatização; severidade de sintomas.

\section{Symptoms Assessment Scale-40 (EAS-40): validity and reliability in a non clinical sample}

\begin{abstract}
The EAS-40 is a 40 items, sef-report scale designed to evaluate psychopathological symptoms and it was adapted from the Symptom Checklist-90-R $(S C L-90-R)$ to Brazilian outpatiens of general hospitals. The aim was to estimate the test-retest reliability and the criterion validity of the EAS-40 to a non clinical population. The sample, composed by II 3 college students ( $99 \mathrm{~F}$ and I4 M), answered to the EAS-40 and to the Brazilian version of the Toronto Alexithymia Scale (TAS) (external criterion). Results suggested that the EAS-40 can be a useful instrument to evaluate colleges' psychological symptoms with good temporal stability. It allows discriminating populations with different profiles. As to the concurrent validity, results showed that the EAS-40 dimensions are diversely correlated to the various TAS components. New researches, involving a more balanced sample concerning to participants' gender must be carried out.
\end{abstract}

Keywords: Psychoticism; anxiety; somatization; symptoms severity.

\section{Escala de Evaluación de Síntomas-40 (EES-40): validez y precisión en muestra no clínica}

\section{Resumen}

La EES-40, una escala de 40 ítems de tipo de auto-relato, objetiva evaluar síntomas psicopatológicos y fue adaptada del Symptom Checklist-90-R (SCL-90-R) para pacientes ambulatoriales brasileños de hospitales generales. El objetivo de la investigación fue estimar la validez de grupos comparados, validez concurrente y precisión test-retest de la EES-40 para una población no clínica. La muestra de II 3 universitarios (99 M y l4 H) respondió la EES\$) y la versión brasileña de la Toronto Alexithymia Scale (TAS) (criterio externo). Los resultados sugieren que la EES-40 puede ser útil para la evaluación de síntomas psicológicos de universitarios con buena estabilidad temporal, permitiendo discriminar poblaciones con perfiles diferentes. En relación a la validez concurrente, las dimensiones de la EES-40 se mostraron correlacionadas de diversas formas con los componentes de la TAS. Nuevas investigaciones deben ser realizadas llevando en cuenta una distribución mejor de las muestras en relación al sexo.

Palabras clave: psicoticismo, ansiedad, somatización, severidad de síntomas. 


\section{Introdução}

Os anos de universidade constituem, para grande parte dos alunos, uma etapa tardia da adolescência e também um momento de novos e variados desafios que demandam novas habilidades e condições psicológicas específicas. Como conseqüência das exigências de desempenho intelectual e de novos papéis sociais, sintomas psicopatológicos podem eventualmente se manifestar como forma ineficaz de adaptação (Simon, 1997). Para a maioria destes jovens, os sintomas não refletem propriamente uma doença ou transtorno mental, mas devem ser adequadamente avaliados em situações de aconselhamento e de orientação psicológicos (Johnson, Ellison \& Heikkinen, 1989).

Instrumentos de avaliação que permitam estimar a severidade de sintomas psicológicos demonstram qualidades psicométricas compatíveis com esta população, podem, portanto, ser muito úteis e mesmo necessários (Todd, Deane \& McKenna, 1997). Dentre os disponíveis em nosso meio e que atendem a estas especificações, encontra-se a Escala de Avaliação de Sintomas-40 (EAS-40) (Laloni, 200I).

A EAS-40 é um instrumento de auto-relato resultante da adaptação em pacientes de hospitais gerais brasileiros proveniente do Symptom Checklist- 90-R (SCL-90-R) (Derogatis, 1994), um dos instrumentos mais utilizados na prática e na pesquisa voltadas para a avaliação de saúde mental. O SCL-90-R é composto de 90 itens destinados à avaliação de sintomas psicopatológicos. As respostas são dadas em escala Likert de cinco pontos, em que o respondente deve indicar o quanto aquele problema o tem preocupado ou angustiado durante os últimos sete dias. Os itens dividem-se segundo nove dimensões sintomáticas: somatização, obsessividade-compulsividade, sensibilidade interpessoal, depressão, ansiedade, hostilidade, ansiedade fóbica, idéias paranóides e psicoticismo e o padrão e a intensidade dos sintomas pode sugerir a presença de transtornos psicológicos (Derogatis, 1994).

A pesquisa de adaptação do SCL-90-R para a população brasileira contou com amostra de 599 pacientes de hospital geral dos quais 200 eram pacientes do ambulatório de saúde mental (Grupo Caso) e 399 de enfermarias diversas (Grupo Controle) (Laloni, 200I). Foram realizados estudos de precisão (análise de consistência interna e teste e re-teste) e de validade (de construto e de critério). Os resultados indicaram boa consistência interna (alfa de Cronbach entre 0,73 e 0,88 ) e estabilidade temporal regular entre 7 e 15 dias ( $r$ entre 0,40 e 0,82 ). $O$ estudo de validade de construto, por meio de análise fatorial, análise de agrupamento e análise de correspondência, resultou em quatro fatores interpretáveis $(40 \%$ da variância total): psicoticismo, obsessividade-compulsividade, somatização e ansiedade. A escala adaptada ficou com 40 itens, sendo dez para cada dimensão.

A análise da sensibilidade dos itens sugeriu a redução da escala Likert de cinco para três pontos correspondentes, respectivamente, a nenhum, um pouco e muito. Apesar desta adaptação contrariar a indicação da literatura de que se devem evitar escalas com menos de cinco pontos (Bordens \& Abbott, 1999), a discriminação de três pontos de severidade dos sintomas costuma ser suficiente para a orientação da intervenção psicológica e médica e reflete, de modo mais fidedigno, a maneira como os pacientes percebem seus sintomas (Laloni, 200I). Como conseqüência, a EAS40 resultou num instrumento de mais fácil aplicação com um número reduzido de itens e respostas, restritas a três alternativas. $O$ mesmo se pode dizer do número de dimensões que passaram de nove para quatro, o que atende de forma mais adequada aos perfis sintomáticos observados na prática clínica (Laloni, 200I).

A EAS-40 foi utilizada por Tombolato (2005) em estudo exploratório e teve como objetivo avaliar a severidade dos sintomas psicopatológicos e a qualidade de vida - medida com o WHOQOL-bref (Fleck e cols, 2000) - de estudantes universitários trabalhadores $(N=122)$, quando comparados a universitários não-trabalhadores $(N=18)$. Ao contrário do esperado, não foram encontradas diferenças significantes entre os dois grupos, em nenhuma das medidas efetuadas. Ao discutir os resultados, a autora levanta a hipótese de que a conciliação de trabalho e estudo nesta população não chega a comprometer a qualidade de vida ou a probabilidade de desenvolvimento de sintomas psicopatológicos devido, possivelmente, aos 
benefícios que este pode ensejar, pois viabiliza economicamente a realização do curso universitário, permite a obtenção de qualificação profissional e pode se constituir em um primeiro degrau para a inserção no mercado de trabalho.

Outras pesquisas, envolvendo amostras clínicas e/ ou pessoas idosas, também utilizaram a EAS-40 como uma das medidas realizadas. Por exemplo, Pregnolatto (2005) utilizou a EAS-40 em pesquisa com pacientes renais crônicos submetidos à hemodiálise; Yoshida (no prelo), em estudo de validade da Toronto Alexithymia Scale - TAS (Taylor, Ryan \& Bagby, 1985), utilizou-a como medida de critério externo; e Khater e Yoshida (2006) empregaram-na para o estudo de sintomas psicopatológicos e qualidade de vida de alunos de uma universidade da terceira idade, comparados a idosos em atendimento ambulatorial de hospital geral.

Apesar de desenvolvida no ambiente hospitalar, supôs-se que a EAS-40 pudesse vir a ser empregada também em outros contextos, necessários a fim de avaliar, de forma rápida e segura, a severidade dos sintomas psicológicos segundo diferentes dimensões. E neste sentido, considerou-se necessário dar continuidade ao processo de identificação das propriedades psicométricas da escala, pesquisando outras medidas de evidência de validade e de precisão, desta feita, junto a um estrato da população não-clínica, e mais especificamente, universitários.

Levantaram-se, assim, como hipóteses exploratórias, que os universitários apresentariam escores médios inferiores aos da amostra clínica do estudo original de Laloni (200I) (validade de grupos comparados), e que haveria associação positiva entre a EAS-40 e uma medida de alexitimia tomada como critério de validade concorrente. A precisão da EAS-40, por meio de teste e reteste, foi também estimada.

\section{Sobre a medida de critério}

Alexitimia corresponde a um construto multidimensional relativo a uma deficiência no processamento das emoções e inclui, dificuldade de identificação e descrição dos próprios sentimentos e emoções; confusão das emoções com sensações corporais; rebaixamento na capacidade de fantasiar; e um estilo cognitivo orientado para o exterior (Taylor,
2000; Taylor \& Bagby, 2004). Dentre os instrumentos de avaliação existentes optou-se pela Toronto Alexithymia Scale (TAS) (Taylor, Ryan \& Bagby, 1985), cuja versão em português demonstrou estrutura fatorial compatível à da original em inglês e à da versão em espanhol, em amostra com universitários brasileiros (Yoshida, 2000). Outras pesquisas, com a mesma versão da TAS, já indicaram a associação de alexitimia com medida de depressão (Maciel \& Yoshida, 2006), neuroticismo (Maciel \& Yoshida, 2006; Yoshida, no prelo), qualidade de vida (Piccoli, 2005) e sintomas psicopatológicos (Pregnolatto, 2005; Yoshida, no prelo).

Ainda que grande parte da literatura sobre alexitimia focalize sua relação com diferentes doenças somáticas como um fator de risco (Taylor, 2000), há autores que defendem que ela é uma característica de personalidade, traduzida em um estilo emocional de enfrentamento, cujo objetivo é o de evitar as experiências emocionais negativas (por ex., Fukunishi \& Rahe 1995 e Stewart, Zvolensky \& Eifert, 2002). De acordo com esta perspectiva, a alexitimia seria uma variável contínua distribuída na população, podendo ou não estar associada a outros distúrbios psicológicos. Há de fato evidências de que em populações nãoclínicas a tendência alexitímica de enfrentamento seja ativada por situações estressantes da vida tais como, por exemplo, a do ingresso na universidade (Kerr, Johson, Gans \& Krumine, 2004), além de estar freqüentemente associada a quadros de depressão, ansiedade-traço e também ansiedade-estado (Berthoz, Consoli, Perez-Diaz \& Jouvent, 1999).

Com base em medidas de alexitimia realizadas com a TAS em diferentes extratos da população, alguns autores argumentam que seus fatores avaliam estratégias de enfrentamento com características diversas. Enquanto os componentes que refletem "habilidade de identificar e descrever sentimentos, distinguindo sentimentos de sensações corporais" e "habilidade para comunicar os sentimentos a outras pessoas" compreendem a vertente afetiva da alexitimia, o componente que mede "a capacidade para fantasiar ou o sonhar acordado" e o que reflete "o pensamento externamente orientado" corresponderiam às dimensões cognitivas da alexitimia (Berthoz e cols, 1999; 
Haviland, Hendryx, Cummings, Shaw \& Macmurray, 1991; Hendryx, Haviland \& Shaw, 1991; Nemiah \& Sifneos, 1970). Esta compreensão tem sido útil para interpretar resultados aparentemente incongruentes e sugere que as várias dimensões teóricas da alexitimia "não convergem para um único fenômeno" (Berthoz e cols, 1999, p.376). Deve-se interpretá-las como um construto multidimensional que integra aspectos emocionais e cognitivos.

A seleção da alexitimia como critério externo para o estudo de validade concorrente da EAS-40 baseouse, portanto, no fato dela ser freqüentemente associada a problemas psicológicos e psiquiátricos, tanto de populações clínicas como não-clínicas (Berthoz e cols., 1999; Fukunishi \& Rahe, 1995; Taylor \& Bagby, 2004). E nesta medida, a expectativa era a de encontrar associação positiva, porém moderada, entre a severidade dos sintomas psicopatológicos e o grau de alexitimia também em estudantes universitários. Quanto à medida de teste e reteste, esperava-se estabilidade temporal das medidas igual ou superior à demonstrada pelos pacientes de hospital geral (Laloni, 200I).

\section{Método}

\section{Participantes}

A amostra foi constituída por 113 estudantes dos cursos de Odontologia (45\%), Fonoaudiologia (28\%) e Ciência Farmacêutica (27\%), de uma universidade particular do interior paulista, com idades entre $19 \mathrm{e}$ 30 anos $(M=21,44 ; D P=2,08)$ e majoritariamente do sexo feminino $(N=99)$.

\section{Instrumento}

Escala de Avaliação de Sintomas - 40 (EAS-40) (Laloni, 200I) - A escala - tipo auto-relato - com 40 itens avalia sintomas psicopatológicos, segundo quatro dimensões: psicoticismo (FI), "um contínuo entre psicose e depressão com sintomas de hostilidade e idéias paranóides" (p. II9); obsessividadecompulsividade (F2), "um conjunto de sintomas de ações e pensamentos repetidos acompanhados de desconforto nas relações interpessoais" (p.l19); somatização (F3), "sintomas comuns aos transtornos somáticos e somatoformes" (p. I 19) e ansiedade (F4), "um conjunto de sintomas desde a ansiedade generalizada até ansiedade fóbica dirigida a situações ou objetos" (p.I 19). A avaliação da intensidade do sintoma é dada por uma escala Likert de três pontos $(0=$ nenhum, I = pouco, 2 = muito). $\mathrm{O}$ escore médio total corresponde ao Índice Global de Severidade IGS, considerado "o melhor indicador simples do nível atual ou intensidade da desordem" (p. 25). O Total de Sintomas Positivos - TSP, obtido pelo somatório de respostas positivas (alternativas I ou 2 na escala Likert), dá "uma medida da abrangência dos sintomas" (p.25). E o Índice de Distúrbio de Sintomas Positivos -IDSP, resultante da divisão do somatório dos itens válidos pelo TSP, fornece "uma medida de estilo de resposta indicando quando o respondente está aumentando ou atenuando os distúrbios psicossomáticos" (p.25). Apesar de se constituírem em índices algo diferentes da sintomatologia psicopatológica dos sujeitos, todos refletem sua severidade, motivo pelo qual, nesta pesquisa, utilizouse apenas o IGS nas análises realizadas.

Toronto Alexithymia Scale/ TAS (Taylor, Ryan \& Bagby, 1985) - A versão em português da TAS (Yoshida, 2000) avalia quatro fatores relacionados à personalidade $e$ expressam a dificuldade em lidar com os sentimentos, emoções e que integram o construto de alexitimia. São elas: (FI) dificuldade em descrever e identificar os sentimentos, distinguindo das sensações corporais; (F2) dificuldade em fantasiar ou sonhar acordado (daydreaming) (F3) preferência por focalizar eventos externos a internos; (F4) dificuldade em expressar e compreender os sentimentos e as emoções. As respostas aos itens são dadas em escala Likert de cinco pontos que variam de (I) discordo inteiramente a (5) concordo plenamente. $O$ escore total fornece o grau de alexitimia. $\mathrm{Na}$ literatura internacional, o ponto de corte para alexitimia é 74, enquanto que escores iguais ou menores que 62 são obtidos por sujeitos não alexitímico (Taylor e cols., 1988). Para os escores entre 63 e 73 não é possível um diagnóstico conclusivo. $\mathrm{Na}$ pesquisa com universitários brasileiros (Yoshida, 2000), o escore médio para a amostra feminina ( $N=$ 394) foi $63,69(D P=10,01)$, para a amostra masculina $(N=187), 62,08(D P=10,19)$ e $63,13(D P=10,12)$ 
para a amostra total $(N=58 \mathrm{I})$. Obteve-se boa consistência interna $(\alpha=0,72)$ e estabilidade no teste e reteste $(r=0,72)$.

\section{Procedimento}

A aplicação dos instrumentos foi realizada em sala de aula pela segunda autora, em horários cedidos pelos professores. Todos os participantes responderam a EAS-40 e a TAS. Para o estudo de precisão de teste e reteste, 26 participantes, sendo três do sexo masculino e 23 do feminino, responderam novamente a EAS-40, após 15 dias, também em salas de aula. A participação foi voluntária e antecedida da assinatura de termo de consentimento livre esclarecido, em que se explicitava os objetivos e condições da pesquisa. $O$ projeto foi aprovado pelo Comitê de Ética da instituição.

\section{Resultados}

A Tabela I apresenta os escores médios e desvios-padrão da EAS-40 da amostra total e de cada um dos cursos, de acordo com o sexo dos participantes. Observa-se que houve uma tendência das mulheres a apresentarem escores médios ligeiramente superiores aos dos homens nos cursos em que houve participantes dos dois sexos, Odontologia e Ciência Farmacêutica. No entanto, o teste $t$ para amostras independentes, aplicado aos Índices Globais de Severidade (IGS) de homens e de mulheres indica que a diferença não é estatisticamente significativa entre os se$\operatorname{xos}[t(I \mid I)=0,33 ; p>0,10]$.

Da mesma forma, não foram encontradas diferenças significativas entre os IGS das mulheres divididas segundo os cursos de Odontologia x Fonoaudiologia, Odontologia x Ciência Farmacêutica e Fonoaudiologia e Ciência Farmacêutica, nem entre os estudantes masculinos de Odontologia e Ciência Farmacêutica $(p>0,10)$ (Tabela 2). Os valores muito semelhantes sugerem a consistência das medidas e as variáveis, sexo e curso, não apresentam relação com a severidade dos sintomas. Apesar disto, foi mantida a separação segundo o sexo nas análises a fim de viabilizar a comparação com os resultados de Laloni (200I), divididos segundo esta variável.

Tabela I. Médias e Desvios Padrão da EAS-40 de acordo com o sexo da amostra geral e por curso.

\begin{tabular}{|c|c|c|c|c|c|c|c|c|c|c|c|c|c|c|}
\hline \multirow{3}{*}{ EAS-40 } & \multicolumn{4}{|c|}{ Geral $(N=113)$} & \multicolumn{4}{|c|}{ Odontologia $(N=51)$} & \multirow{2}{*}{\multicolumn{2}{|c|}{$\begin{array}{c}\text { Fonoaudiologia } \\
\qquad(N=32) \\
\text { Feminino } \\
(N=31)\end{array}$}} & \multicolumn{4}{|c|}{$\begin{array}{l}\text { Ciência Farmacêutica } \\
\qquad(N=30)\end{array}$} \\
\hline & \multicolumn{2}{|c|}{$\begin{array}{l}\text { Masculino } \\
(N=14)\end{array}$} & \multicolumn{2}{|c|}{$\begin{array}{l}\text { Feminino } \\
(N=99)\end{array}$} & \multicolumn{2}{|c|}{$\begin{array}{l}\text { Masculino } \\
\qquad(N=8)\end{array}$} & \multicolumn{2}{|c|}{$\begin{array}{l}\text { Feminino } \\
(N=43)\end{array}$} & & & \multicolumn{2}{|c|}{$\begin{array}{l}\text { Masculino } \\
\qquad(N=5)\end{array}$} & \multicolumn{2}{|c|}{$\begin{array}{l}\text { Feminino } \\
(N=25)\end{array}$} \\
\hline & M & DP & M & DP & M & DP & M & DP & M & DP & M & DP & M & DP \\
\hline IGS & 0,55 & 0,40 & 0,60 & 0,41 & 0,58 & 0,36 & 0,63 & 0,42 & 0,51 & 0,37 & 0,49 & 0,40 & 0,65 & 0,46 \\
\hline F 1 & 0,56 & 0,34 & 0,59 & 0,42 & 0,59 & 0,24 & 0,61 & 0,38 & 0,50 & 0,40 & 0,54 & 0,51 & 0,68 & 0,51 \\
\hline $\mathrm{F} 2$ & 0,77 & 0,40 & 0,76 & 0,40 & 0,84 & 0,37 & 0,80 & 0,36 & 0,64 & 0,34 & 0,76 & 0,48 & 0,85 & 0,51 \\
\hline F3 & 0,53 & 0,50 & 0,55 & 0,42 & 0,50 & 0,56 & 0,55 & 0,49 & 0,53 & 0,40 & 0,56 & 0,51 & 0,56 & 0,31 \\
\hline $\mathrm{F} 4$ & 0,34 & 0,37 & 0,49 & 0,41 & 0,39 & 0,26 & 0,57 & 0,47 & 0,37 & 0,36 & 0,10 & 0,10 & 0,51 & 0,51 \\
\hline
\end{tabular}

* No curso de fonoaudiologia houve apenas I participante do sexo masculino

Tabela 2. Valores de $t$ (para amostras independentes) da EAS-40 entre cursos, para cada sexo.

\begin{tabular}{ccc}
\hline Curso & \multicolumn{2}{c}{$t$} \\
\cline { 2 - 3 } & Masculino & Feminino \\
\hline Odontologia x Fonoaudiologia & - & 1,27 \\
Odontologia x Ciência Farmacêutica & 0,43 & 0,18 \\
Fonoaudiologia x Ciência Farmacêutica & - & 1,28 \\
\hline
\end{tabular}




\section{Precisão de teste e reteste}

Os coeficientes de correlação de Pearson variaram entre 0,80 e 0,93 , como se segue: 0,93 (IGS), 0,80 (FI), 0,84 (F2), 0,87 (F3) e 0,9I (F4), indicando boa estabilidade temporal tanto da escala total quanto em relação às suas quatro dimensões.

\section{Validade}

Para o estudo de validade de grupos comparados, os resultados da EAS-40 foram confrontados com os de um grupo de pacientes de ambulatório de saúde mental (Grupo Caso, $N=200$ ) (Laloni, 200I). Conforme o esperado, os resultados apontaram médias inferiores para o IGS e para as quatro dimensões da EAS-40 na amostra estudantil, tanto para os sujeitos do sexo feminino quanto do masculino (Tabela 3). como critério (Tabela 4). Considerando que o tamanho das amostras costuma exercer grande influência sobre os coeficientes de correlação, apenas os resultados das amostras feminina e masculina são apresentados, sem reuni-los numa amostra geral, dado que sofreria o viés imposto pelo predomínio de mulheres na amostra.

Obteve-se correlação significativa $(p<0,05)$, porém modesta, para a amostra feminina entre 0 IGS, FI e F2 da EAS-40 e o escore total da TAS (Tabela 4). As correlações foram igualmente significativas entre os escores totais e parciais da EAS-40 e os de FI da TAS. Na amostra masculina, apenas o FI da EAS-40 apresentou correlação significativa com o escore total e o FI da TAS. E, contrariamente ao esperado, obteve-se correlações negativas significativas na amostra feminina

Tabela 3. Média, Desvios-Padrão e valores de $t$ (amostras independentes) da EAS-40 de amostra clínica (Estudo I, $N=200$ ) e de universitários (Estudo 2, $N=|| \mid 3$ ), de acordo com o sexo.

\begin{tabular}{|c|c|c|c|c|c|}
\hline \multirow{3}{*}{ Fator } & \multirow{2}{*}{\multicolumn{2}{|c|}{$\begin{array}{c}\text { Estudo } 1 \\
\text { Mulher ( } \mathrm{N}=137)\end{array}$}} & \multirow{2}{*}{\multicolumn{2}{|c|}{$\begin{array}{c}\text { Estudo } 2 \\
\text { Mulher }(\mathrm{N}=99)\end{array}$}} & \multirow{3}{*}{$t$} \\
\hline & & & & & \\
\hline & $M$ & $\mathrm{DP}$ & $M$ & $\mathrm{DP}$ & \\
\hline $\mathrm{F} 1$ & 1,15 & 0,83 & 0,59 & 0,42 & $6,22^{* * *}$ \\
\hline $\mathrm{F} 2$ & 1,59 & 0,96 & 0,76 & 0,40 & $8,30^{* * *}$ \\
\hline $\mathrm{F} 3$ & 1,62 & 0,99 & 0,55 & 0,42 & $6,66^{* * *}$ \\
\hline $\mathrm{F} 4$ & 1,53 & 1,00 & 0,49 & 0,41 & $10,40^{* * *}$ \\
\hline IGS & 1,47 & 0,94 & 0,60 & 0,41 & $8,7^{* * *}$ \\
\hline \multirow{2}{*}{ Fator } & \multicolumn{2}{|c|}{ Homem $(\mathrm{N}=63)$} & \multicolumn{2}{|c|}{ Homem $(\mathrm{N}=14)$} &  \\
\hline & $M$ & $\mathrm{DP}$ & $M$ & $\mathrm{DP}$ & $t$ \\
\hline F1 & 1,20 & 0,82 & 0,56 & 0,34 & $2,91^{*}$ \\
\hline F2 & 1,51 & 0,96 & 0,77 & 0,40 & $2,84^{*}$ \\
\hline F3 & 1,34 & 0,91 & 0,53 & 0,50 & $3,24^{* *}$ \\
\hline F4 & 1,41 & 0,99 & 0,34 & 0,37 & $3,96^{* * *}$ \\
\hline IGS & 1,36 & 0,92 & 0,55 & 0,40 & $3,24^{* * *}$ \\
\hline
\end{tabular}

Estudo I, Laloni (200I), Estudo 2, o atual; $t=$ Teste $\mathrm{t}$ (amostras independentes)

* $\mathrm{p}<0,01 ;{ }^{* *} \mathrm{p}<0,002 ; * * * * \mathrm{p}<0,001$

Para a validade concorrente foram estimados coeficientes de correlação de Pearson, entre os escores totais e parciais da EAS-40 e da TAS, utilizada entre o F2 da EAS-40 e o F2 da TAS; entre o F3 da EAS-40 e o F3 da TAS; e entre o FI da EAS-40 e o F2 da TAS. 
Tabela 4. Correlação entre TAS e EAS-40 de acordo com o sexo.

\begin{tabular}{ccccccc}
\hline \multirow{2}{*}{ Sexo } & TAS & \multicolumn{5}{c}{ EAS-40 } \\
\cline { 3 - 7 } & Total & IGS & F 1 & F2 & F3 & F4 \\
\hline \multirow{2}{*}{ Feminino } & F 1 & $0,248^{* *}$ & $0,243^{* *}$ & 0,066 & 0,106 \\
$(N=99)$ & F 2 & $-0,193$ & $-0,195$ & $-0,278^{* * *}$ & $-0,050$ & $-0,102$ \\
& F 3 & $-0,067$ & $-0,030$ & $-0,104$ & 0,005 & $-0,096$ \\
& F 4 & $-0,066$ & $-0,021$ & 0,032 & $-0,230^{*}$ & 0,005 \\
& Total & 0,415 & $0,569^{*}$ & 0,297 & 0,202 & 0,351 \\
Masculino & F 1 & 0,480 & $0,658^{* *}$ & 0,343 & 0,190 & 0,192 \\
$(N=14)$ & F 2 & $0,574^{*}$ & 0,443 & 0,363 & 0,430 & $0,582 *$ \\
& F 3 & $-0,112$ & $-0,095$ & $-0,243$ & 0,110 & 0,400 \\
& F 4 & $-0,007$ & 0,214 & 0,390 & $-0,359$ & $-0,418$ \\
\hline
\end{tabular}

*p $<0,05 ; * * \mathrm{p}<0,02 ; * * * \mathrm{p}<0,001$

\section{Discussão}

As médias femininas, ligeiramente superiores às masculinas na amostra geral, divididas segundo os cursos, não chegaram a ser suficientemente expressiva para mostrar maior severidade dos sintomas psicopatológicos entre universitárias quando comparadas aos universitários $(t, p>0,10)$ (Tabela I). Estes dados coincidem com os de Pregnolatto (2005) que não encontrou diferença na intensidade dos sintomas entre pacientes com insuficiência renal crônica, divididos de acordo com o sexo, ainda que as mulheres também tenham apresentado escores médios ligeiramente superiores aos dos homens. Quanto ao estudo de Laloni (200I), as mulheres do Grupo Caso se diferenciaram dos homens na intensidade dos sintomas nas dimensões Somatização e Depressão. No entanto, é preciso notar que estes dados se referem às dimensões e aos itens originais do SCL-90-R, o que requer cautela nas comparações.

Em relação aos escores médios das dimensões, observa-se que os mais elevados foram os da dimensão obsessividade-compulsividade, coincidindo com os achados do estudo de Pregnolatto (2005) e também com os de Tombolato (2005). Eles compararam universitários que trabalham com universitários que não trabalham. As mulheres também apresentaram índices ligeiramente superiores aos homens.

Os estudos até o presente sugerem, portanto, não haver diferenças nas intensidades de sintomas quando se comparam homens e mulheres, seja em amostras clínicas ou não-clínicas. Quando se comparou grupo do mesmo sexo, mas de cursos diferentes não foram também observadas diferenças (Tabela 2). Estes dados, portanto, podem ser interpretados como evidência de consistência interna do instrumento.

Em relação à precisão de teste e reteste, a EAS40 revelou excelente estabilidade temporal entre universitários ( $r$ entre 0,80 e 0,93, 15 dias) quando comparados aos da amostra clínica (entre sete e 15 dias) que ficaram entre 0,40 e 0,82 (Laloni, 200I). O perfil não-clínico da amostra e também a faixa etária dos participantes contribuíram provavelmente para este efeito.

As diferenças entre as amostras, clínica e não-clínica, foram corroboradas pela análise das diferenças entre os escores médios dos universitários e os do Grupo caso de Laloni (200I) (Tabela 3). Para os dois sexos, as médias dos universitários foram significantemente inferiores às da população de pacientes. Estes resultados podem ser interpretados como evidências da capacidade da EAS-40 de discriminar populações com diferentes níveis de sintomas psicopatológicos e, portanto, apontam para a 
especificidade (capacidade de classificar indivíduos não-doentes) e para a sensibilidade (capacidade de classificar como doentes os doentes verdadeiros) da escala, constituindo-se indicadores relevantes de sua validade.

No estudo da validade concorrente (Tabela 4), obteve-se, na amostra feminina, coeficientes significativos de correlação, porém moderados, entre o IGS e o escore total da TAS, e também entre o IGS e o FI da TAS, explicados, respectivamente, por $4 \%$ e 17,6\% da variância. $\mathrm{Na}$ amostra masculina, estas correlações não chegaram a ser significativas, apesar dos coeficientes indicarem associação positiva entre as variáveis em questão.

Procurando interpretar estes resultados, vale notar que $\circ \mathrm{FI}$ da TAS, que mede dificuldade de descrever os sentimentos, é considerado o fator mais representativo do construto alexitimia; seja quando se leva em conta a etimologia da palavra que designa literalmente "falta de palavras para emoções" (Taylor, 2000), seja quando se considera o peso deste fator na explicação da variância total (I7,92\% da versão utilizada) (Yoshida, 2000). Isto é, o escore total da TAS reflete em grande parte a medida de FI, cuja estrutura tem sido reproduzida nas diferentes versões da escala adaptada para vários idiomas, ao contrário dos demais fatores, para os quais diferentes combinações de itens têm sido observadas (Yoshida, no prelo). Pode-se concluir, portanto, que a severidade dos sintomas psicopatológicos entre universitárias encontra-se associada à alexitimia em geral e, mais especificamente, à dificuldade de expressão verbal das emoções, medida pelo FI. Quanto aos universitários não é possível afirmar nada com base nos atuais resultados.

Em relação aos demais fatores da TAS, o IGS se correlacionou positivamente de forma significativa apenas com o F2 da TAS na amostra masculina (33\%), sugerindo que quanto mais intensa a sintomatologia psicopatológica entre universitários mais reduzida a tendência para "sonhar acordado". Esta característica é congruente com a expectativa teórica de que os conflitos psicopatológicos mobilizam mecanismos de defesa que resultam em inibições de diferentes ordens, entre as quais se encontra a capacidade de fantasiar. Por outro lado, na amostra feminina, o coefici- ente foi surpreendentemente negativo e não significativo (3,7\%), não permitindo qualquer ilação a seu respeito, salvo alguma especificidade da amostra masculina que teria agido como confounding. Com base nos resultados deste estudo, pode-se dizer que apenas em homens universitários a capacidade de fantasiar e de sonhar acordado fica prejudicada com o aumento dos sintomas psicopatológicos.

Quanto à dimensão psicoticismo (FI), as correlações foram significativas com os escores totais da TAS e com os respectivos FI da TAS, para homens e mulheres. A dimensão psicoticismo foi definida por Laloni (200 I) como "representando um contínuo entre psicose e depressão com sintomas de hostilidade e idéias paranóides" (p.l19). É a dimensão da EAS40 , associada a sintomas patológicos mais severos e, por conseguinte, era de fato esperado que estivesse associada à dificuldade de verbalizar e descrever sentimentos e emoções, independente do sexo. Interessante ainda notar que os coeficientes masculinos $(32,4 \%$ e $43,3 \%)$ foram bastante superiores aos das mulheres $(6,1 \%$ e $20,7 \%)$.

A dimensão obsessividade-compulsividade (F2) apresentou correlação positiva com o escore total e FI da TAS apenas na amostra feminina e, explicadas, respectivamente, por $5,9 \%$ e $14 \%$ da variância. Esta dimensão é definida como, "um conjunto de sintomas, pensamentos e ações, repetidos acompanhados de desconforto nas relações interpessoais" (Laloni, 200I, p.I 19). E nesta medida, era também esperado que estas características fossem acompanhadas de dificuldades na expressão de emoções.

De forma paradoxal, na amostra feminina, a obsessividade-compulsividade (F2) correlacionou-se negativamente $(7,7 \%)$ com o F2 da TAS. Trata-se de uma relação aparentemente inesperada posto que os enunciados dos itens refletem uma "sujeição" do indivíduo ao "seu mundo interno", levando-o a executar tarefas de forma compulsória e a exercer controle sobre suas emoções. E nesta medida, um aumento na intensidade dos sintomas deveria se acompanhado de um empobrecimento da capacidade de fantasiar e não o contrário.

Mas se se considera a proposta de que "a capacidade para fantasiar ou o sonhar acordado" reflete uma 
das dimensões cognitivas da alexitimia (Berthoz e cols., 1999; Haviland e cols., 1991; Hendryx e cols., 199I; Nemiah \& Sifneos, 1970), é possível cogitar que frente a sintomas que impõem um desconforto ao sujeito, por levá-lo a repetir tarefas já realizadas, ou ainda que o impelem a controlar suas emoções, o sonhar acordado estaria servindo como uma forma de enfrentamento, na medida em que permitiria "certa liberdade" para experienciar situações e emoções que não podem ser expressas externamente. $E$, neste sentido, esta dimensão da alexitimia estaria refletindo uma estratégia adaptativa para fazer face ao sofrimento. Esta suposição precisa, todavia, ser ratificada por novas observações, sendo mais cauteloso afirmar-se, por ora, que as correlações negativas permanecem incertas.

A dimensão somatização (F3) apresentou, na amostra feminina, correlação positiva e significativa com o FI da TAS (explicada por 4,4\%), corroborando o pressuposto de que os sintomas dos transtornos somáticos e somatoformes são gralmente associados a características alexitímicas. Isto é, frente à impossibilidade de descrever e identificar verbalmente as emoções, estas seriam vividas como "dores" ou "males" do corpo (Berthoz e cols., 1999).

Contrastando com estas evidências, há as correlações inversas da somatização (F3) com o F4 da TAS $(5,3 \%)$. Trata-se de dado contraditório posto que o F4 da versão em português da TAS (que corresponde ao F2 da versão original) é considerado, juntamente com o $\mathrm{FI}$, um componente ligado à vertente emocional da alexitimia (Berthoz e cols., 1999; Haviland e cols., 1991; Hendryx e cols., 1991; Nemiah \& Sifneos, 1970). Assim, era esperado que acompanhasse o mesmo comportamento de FI, correlacionandose positivamente com a somatização. É provável que um viés de ordem psicométrica desta dimensão da EAS-40 ou do F4 da TAS, ou de ambos os instrumentos, tenha contribuído para este resultado. Há, portanto, necessidade de se obter novas evidências para que se possa identificar melhor se se trata de uma questão das medidas, ou uma especificidade da amostra estudada.

E finalmente, a dimensão da ansiedade (F4) da EAS40 apresentou correlação significativa apenas com o fator um (FI) da TAS, na amostra feminina (I I,3\%), coincidindo com os resultados de Berhoz e cols. (1999) que realizaram pesquisa com universitárias. Os autores também só encontraram correlação significativa entre medidas de ansiedade-traço e ansiedade-estado e o FI da TAS. Frente a estes resultados, eles questionam se dificuldades em identificar e comunicar sentimentos não estariam "intrinsicamente presentes em diferentes patologias" (p. 377).

Esta hipótese parece bastante plausível considerando que o $\mathrm{FI}$ da TAS foi o único componente que se correlacionou com todas as dimensões, ao menos na amostra feminina. Além disto, ela é corroborada pelos resultados de Yoshida (no prelo) que também só obteve correlações significativas entre a EAS-40 (total e segundo cada dimensão) e o FI da TAS, em pacientes de hospital geral.

\section{Considerações Finais}

Os resultados sugerem que a EAS-40 pode ser um instrumento útil para a avaliação de sintomas psicológicos junto a universitários, pois detém boa consistência interna, estabilidade temporal e permite discriminar populações com perfis psicopatológicos diferentes. Quanto ao estudo de validade de critério, ela mostrou que as dimensões da EAS-40 são correlacionadas de formas diversas com os componentes da alexitimia. E que, especificamente, para as universitárias, a intensidade de sintomas psicopatológicos encontra-se associada ao nível de alexitimia devido, principalmente, à dificuldade de descrever e identificar os sentimentos, distinguindo-os das sessões corporais.

Apesar de serem necessárias novas pesquisas de validade e de precisão, incluindo estudos da estrutura fatorial da escala, os resultados sugerem que a EAS40 pode ser utilizada em programas de cuidados à saúde mental de universitários. Efetivamente, ela permite obter em programas de orientação e seguimento de universitários, avaliações rápidas, compreensivas e sistemáticas sobre quais estudantes precisam de atenção especial ou de encaminhamento para psicoterapia. A exemplo do SCL-90-R, utilizado em 
processos de aconselhamento de estudantes na Universidade de Wisconsin - Madison (Johnson, Ellison, \& Heikkinen, 1989), a EAS-40 pode ajudar na identificação de situações de crise, na indicação do tipo mais adequado de acompanhamento a ser dado a cada caso, na identificação do foco do atendimento, ou ainda, por meio de avaliações sucessivas, fornecer evidências da eficiência do aconselhamento ou da orientação prestados.

Os resultados desta pesquisa, ainda que preliminares, sugerem, portanto, que a EAS-40 é um instrumento que deve ser mais pesquisado enquanto uma ferramenta auxiliar em processos de avaliação psicológica de populações clínicas e não-clínicas. E neste sentido, deve-se procurar conhecer as propriedades psicométricas deste instrumento junto a outros substratos da população em geral, de forma a viabilizar, entre outras coisas, o desenvolvimento de normas brasileiras.

\section{Referências}

Berthoz, S., Consoli, S., Perez-Diaz, F., \& Jouvent, R. (1999). Alexithymia and anxiety: compounded relationships? A psychometric study. European Psychiatry, 14, 372-378.

Bordens, K. S., \& Abbott, B. B. (1999). Research Design and Methods: a process approach (4th ed.). Mountain View: Mayfield Publishing Company.

Derogatis, L. R. (1994). Symptom Checklist-90-R (SCL-90-R) Administration, Scoring, and Procedures Manual (3rd ed.). Mineapolis: National Computer Systems.

Fleck, M. P. A., Lousada, S., Xavier, M., Chachamovich, E., Vieira, G., Santos, L., \& Pinzon, V. (2000). Aplicação da versão em português do instrumento WHOQOL-bref. Revista de Saúde Pública, 34, 178-183.

Fukunishi, I., \& Rahe, R. H. (1995). Alexithymia and coping with stress in healthy persons: Alexithymia as a personality trait is associated with low social support and poor responses to stress. Psychological Reports, 76, I299-1 304.

Haviland, M. G., Hendryx, M. S., Cummings, M. A., Shaw, D. G., \& Macmurray, J. P. (199I). Multidimensionality and state dependency of alexithymia in recent sober alcoholics. Journal of Nervous \& Mental Disease, 179, 284-290.
Hendryx, M. S., Haviland, M. G., \& Shaw, D. G. (I99I). Dimensions of alexithymia and their relashionships to anxiety and depression. Journal of Personality Assessment, 56, 227-237.

Johnson, R. W., Ellison, R. A., Heikkinen, C. A. (1989). Psychological Symptoms of Counseling Center Clients. Journal of Counseling Psychology, 36, I I0-I I 4.

Khater, E., \& Yoshida, E. M. P. (2006) Alexitimia e sintomas psicopatológicos em pessoas idosas. I Simpósio Internacional de Pesquisa em Psicoterapia, Pontifícia Universidade Católica de Campinas. Trabalhos e Resumos, 17-26.

Kerr, S., Johnson, V. K., Gans, S. E., \& Krumine, J. (2004). Prediciting adjustment during the transition to college: alexithymia, perceived stress, and psychological symptoms. Journal of College Student Development, 45, 593- 6 I I.

Laloni, D. T. (200 I). Escala de Avaliação de Sintomas-90-R SCL90-R: adaptação, precisão e validade. Tese de Doutorado não publicada, Pontifícia Universidade Católica de Campinas, Campinas.

Maciel, M. J. N., \& Yoshida, E. M. P. (2006). Avaliação de alexitimia, neuroticismo e depressão em dependentes de álcool. Revista Avaliação Psicológica, 5, 43-54.

Nemiah, J. C., \& Sifneos, P. E. (1970). Psychosomatics illness: a problem in communication. Psychotherapy and Psychosomatics, 18, $154-160$.

Piccoli, A. P. B. (2005). Qualidade de vida e alexitimia em estudantes de cursos técnicos. Dissertação de Mestrado não publicada, Pontifícia Universidade Católica de Campinas, Campinas.

Pregnolatto, A. P. F. (2005). Alexitimia e sintomas psicopatológicos em pacientes com insuficiência renal crônica. Dissertação de Mestrado não publicada, Pontifícia Universidade Católica de Campinas, Campinas.

Simon, R. (1997). Proposta de redefinição da EDAO (Escala Diagnóstica Adaptativa Operacionalizada). Boletim de Psicologia, XLVII ( 107), 85-94.

Stewart, S. H., Zvolensky, M. J., \& Eifert, G. H. (2002). The relations of anxiety sensitivity, experiential avoidance, and alexithymic coping to young adults' motivations for drinking. Behavior Modification, 26, 274-296.

Taylor, G. J. (2000). Recent developments in alexithymia theory and research. Canadian Journal of Psychiatry, 45, I 34- I 42.

Taylor, G. J., \& Bagby, R. M. (2004). New trends in alexithymia research. Psychotherapy and Psychosomatics, 73, 68-77. 
Taylor, G. J., Bagby, R. M., Ryan, D. P., Parker, J. D., Doody, K. F., \& Keefe, P. (1988). Criterion validity of the Toronto Alexithymia Scale. Psychosomatic Medicine, 50, 500-509.

Taylor, G. J., Ryan, D., \& Bagby, R. M. (1985). Toward the development of a new self-report alexithymia scale. Psychotherapy and Psychosomatics, 44, 191-199.

Todd, D. M., Deane, F. P., \& McKenna, P. A. (I997). Appropriateness of the SCL-90-R adolescent and adult norms for outpatient and nonpatient college students. Journal of Counseling Psychology, 44, 294-301.
Tombolato, M. C. R. (2005). Qualidade de vida e sintomas psicopatológicos do estudante universitário trabalhador. Dissertação de Mestrado não publicada, Pontifícia Universidade Católica de Campinas, Campinas.

Yoshida, E. M. P. (2000). Toronto Alexthymia Scale-TAS: precisão e validade da versão em português. Psicologia: Teoria e Prática, 2, 59-74.

Yoshida, E. M. P. (no prelo). Validade da versão em português da Toronto Alexithymia Scale- TAS para população clínica. Psicologia: Reflexão e Crítica.

Recebido em: 10/08/2006

Revisado em: 19/12/2006

Aprovado em: 12/06/2007

Sobre as autoras:

Elisa Medici Pizão Yoshida (eyoshida.tln@terra.com.br) é Docente do Programa de Pós-Graduação em Psicologia e da faculdade de Psicologia da Pontifícia Universidade Católica de Campinas.

Fernanda Robert de Carvalho Santos Silva (frobert_c@yahoo combr) é bolsista PIBIC-CNPq da Pontifícia Universidade Católica de Campinas.

Endereço para correspondência Pontifícia Universidade Católica de Campinas Programa de Pós-Graduação em Psicologia Av. John Boyd Dunlop, $s / n^{\circ}$ - Jardim Ipaussurama I 30 I 2-970 - Campinas - SP 